\title{
Observation of Variations in Cosmic Ray Showers During Thunderstorms and Implications for Large-Scale Electric Field Changes
}

\section{Rasha Abbasi ${ }^{a, *}$ on behalf of the Telescope Array Collaboration}

(a complete list of authors can be found at the end of the proceedings)

${ }^{a}$ Department of Physics, Loyola University Chicago, Chicago, U.S.A.

E-mail: rabbasi@luc.edu

\begin{abstract}
This work presents the first observation by the Telescope Array Surface Detector (TASD) of the effect of thunderstorms on the development of the cosmic ray showers. Observations of variations in the cosmic ray showers, using the TASD, allows us to study the electric field inside thunderstorms on a large scale without dealing with all the limitation of narrow exposure in time and space using balloons and aircraft detectors. In this work, observations of variations in the cosmic ray shower intensity $(\Delta N / N)$ using the TASD, was studied and found to be on average at the $(1-2) \%$ level. These observations where found to be both negative and positive in polarity. They were found to be correlated with lightning but also with thunderstorms. The size of the footprint of these variations on the ground ranged from (4-24) $\mathrm{km}$ in diameter and lasted for $10 \mathrm{~s}$ of minutes. Dependence of $(\Delta N / N)$ on the electric field inside thunderstorms, in this work, is derived from CORSIKA simulations.
\end{abstract}

$37^{\text {th }}$ International Cosmic Ray Conference (ICRC 2021)

July 12 th - 23rd, 2021

Online - Berlin, Germany

${ }^{1}$ (a complete list of authors can be found at the end of the proceedings)

${ }^{*}$ Presenter 


\section{Introduction}

Understanding lighting initiation is one of the most important questions in atmospheric physics. The heart of the problem of understanding lightning initiation is that, with decades of electric fields measurements, the observed values of detected electric field are not sufficient to create a leader or a stroke propagating on a kilometer(s) scale $[16,18]$. This could mean that either our understanding of how lightning is initiated, or that electric field measurements in thunderstorms, are inaccurate.

Traditionally, balloons and planes are used to make such measurements. However, there are limitations to obtaining such observations. At first, sending planes, balloons, and launching rockets inside thunderstorms can be quite difficult and dangerous. Moreover, thunderstorms scales can span up to kilometers square in size, while the electric field measured by airplanes and balloons spans a small region in comparison. In addition, to be in the right location at the right time where the electric field and the potential difference is of a high value can be of low probability. Most importantly, the instrument sent inside a thunderstorm might be responsible for discharging the thunderstorm itself before the electric field has the chance to build up.

When cosmic ray particles interact in the atmosphere, they produce a shower of secondary particles. During thunderstorms, these showers of secondary particles would accelerate or decelerate depending on their charge and magnitude of the electric field they are propagating through. In principle, studying the effect of the electric field on these secondary particles would allows us to measure and model the electric field in their path indirectly and on a larger scale than direct measurements.

The effect of thunderstorms on the extensive air showers is a hot topic that has been reported on by multiple experiments starting with the Baskan group in 1985 [4]. They argued the effect of the observed cosmic ray variations in the hard and soft component of the shower is due to the electric field in the atmosphere. They also demonstrated a quadratic effect in the muon variation which is independent of the electric field sign. Several studies and observations have followed EAS-TOP [8],Tibet AS [17], ARGO-YBJ [5], SEVAN [13], and Mount Norikura [15], reporting on the cosmic ray secondary showers (electrons, gamma rays, muons, and neutrons) variation in correlation with thunderstorms. Most recently, an indirect measurement of the electric field and the potential difference inside the storm have already allowed the measurement of a greater than one GV potential difference inside the cloud ( predicted by C.T.R. Wilson 90 years ago [7]) by the Grapes Muon Telescope-III scientists [11]. Note that, such potential difference is almost an order of magnitude larger than the previously reported maximum potential in balloon sounding (0.13 GV) [14].

In this work, we will present the effect of the electric field in thunderstorms on the extensive air showers as observed by the Telescope Array Surface Detector (TASD). We will report on the observations in the variation of the cosmic ray extensive shower rate during thunderstorms as the thunderstorm progresses on top of the $700 \mathrm{~km}^{2}$ detector. To interpret this variation, the effect of the electric field in thunderstorms is simulated for multiple simple models. The corresponding increase/decrease of the rate variation in correlation with the modeled electric field is discussed. 

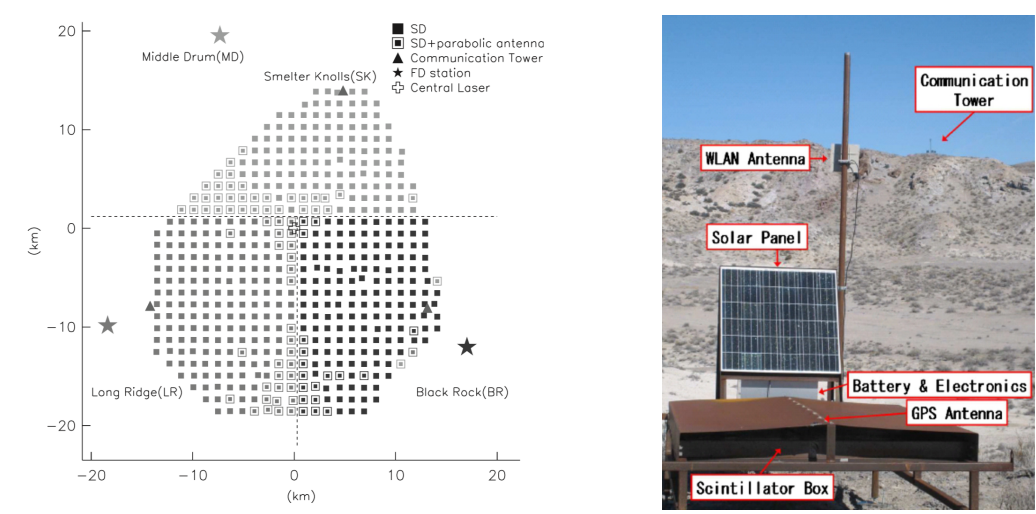

Figure 1: Left: The Telescope Array, consisting of 507 scintillator Surface Detectors (SDs) on a $1.2 \mathrm{~km}$ grid over a $700 \mathrm{~km}^{2}$ area (black squares), and three Fluorescence Detectors MDFD, LRFD, BRFD (stars). Right: A single SD in field. The figure shows the SD's multiple components including a solar panel, a stainless steel box placed under the solar panel housing the battery and the front-end electronics, and a 2.4 $\mathrm{GHz}$ wireless LAN modem communication antenna transmitting data to communication towers surrounding the detector [2].

\section{The Telescope Array Detector}

The Telescope Array (TA) detector is located in the southwestern desert of the State of Utah about $1400 \mathrm{~m}$ above sea level. Currently it is the largest Ultra High Energy Cosmic Ray (UHECR) experiment in the Northern Hemisphere. The TA detector is comprised of Surface Detectors (SDs) surrounded by three Fluorescence Detectors (FDs). The main goal of the TA detector is to explore the origin of UHECRs using their energy, composition, and arrival direction.

The Surface Detector utilizes plastic scintillators to observe the EAS footprint produced by primary cosmic ray interactions in the atmosphere. The Surface Detector array (SD) part of the TA experiment, is composed of 507 scintillator detectors on a $1.2 \mathrm{~km}$ square grid covering $700 \mathrm{~km}^{2}$ in area shown in Figure 1. The scintillator layers and stainless-steal plate are housed in a $1.5 \mathrm{~mm}$ thick box made of stainless steel (top cover is $1.5 \mathrm{~mm}$ thick, with a $1.2 \mathrm{~mm}$ thick bottom). Each detector unit also has a $1 \mathrm{~m}^{2}$ solar panel, a stainless steel box placed under the solar panel housing the battery and the front-end electronics, and a $2.4 \mathrm{GHz}$ wireless LAN modem communication antenna transmitting data to communication towers surrounding the detector. The SD detector is divided into three subparts (The Long Ridge (LR), Black Rock (BR), and Smelter Knolls (SK) subarrays) each denoted by a triangle in Figure 1 and is controlled and send information to its own communication tower. The SD has nearly a $100 \%$ duty cycle [2].

Each surface detector houses two $1.5 \mathrm{~m} \times 1 \mathrm{~m}$ scintillator layers that are $1.2 \mathrm{~cm}$ thick. Each plastic scintillator slab has grooves that has 104 WaveLength-Shifting (WLS) fibers running through them collecting light into PMTs they are bundled and connected to. Plastic scintillators are sensitive to all charged particles. These scintillator layers are separated by a $1 \mathrm{~mm}$ stainless-steal plate.

There are a total of three trigger data levels. Level-0, Level-1, and Level-2. Charged particles triggering the detector with an energy above approximately 0.3 Minimum Ionizing Particle (MIP) are stored in a memory buffer on CPU board as Level-0 trigger data (trigger rate is approximately $750 \mathrm{~Hz}$ ). Charged particles triggering the detector with an energy above approximately 3 MIPs are 
stored as a level-1 trigger event (trigger rate is approximately $30 \mathrm{~Hz}$ ). When three adjacent detectors trigger with an energy above 3 MIPs within $8 \mu$ seconds the data is saved as Level-2 trigger (trigger rate is approximately $0.01 \mathrm{~Hz}$ ). Level-2 trigger is used to study cosmic rays at energies above $10^{19}$ $\mathrm{eV}$. Level-0 main goal is to monitor the health of the detector and is used in this work to study cosmic rays between $\sim 2 \times 10^{10}-10^{13} \mathrm{eV}$.

\section{Observations}

The Telescope Array detector has been in operation since 2008. For data collected between 2008-2019, the TASD observed secondary shower rate was scanned for variations in the cosmic ray secondary showers $(\Delta N / N)$ in correlation with thunderstorms. Due to the complex nature and structure of thunderstorms we have chosen to focus on energetic thunderstorms. As a start we are investigating thunderstorms including a high recorded peak currents $(>90 \mathrm{kA})$ by the National Lightning Detection Network (NLDN). Several thunderstorms where observed to produce a variation in the secondary cosmic ray showers $(\Delta N / N)$.

For example, on September 27 2014, a variation in the intensity of the secondary air showers was observed by the TASD. See Figure 2. In Figure 2. One can see a clear movement of a deficit and an excess in the intensity variation in correlation with a thunderstorm in 10 minutes interval. The direction of the deficit and excess matched lightning events reported by the NLDN but also the direction of movement of the thunderstorm in radar images going from south-west to the north-east. The variations were not correlated with temperature changes at the ground level. It lasted for tens of minutes across the detector. In some cases it was correlated with lightning but in other frames in was correlated with the thunderstorm itself. The size of the variation varies from $6-24 \mathrm{~km}$ on the ground. The variations were observed in excess and deficit modes between $\pm(1-2) \%$.

\section{CORSIKA Simulations}

The simplest electric field structures that can reproduce the main features in the observed secondary shower variations $(\Delta N / N)$ observed by the TASD are.

1. Model I: A uniform electric field layer inside a cloud with a thickness of $2 \mathrm{~km}$ and a distance of $2 \mathrm{~km}$ between the bottom layer of the cloud and the ground level.

2. Model II: A cloud-ground uniform electric field layer. The height of the layer is $2 \mathrm{~km}$ from the ground level $(\sim 1400 \mathrm{~m})$.

While thunderstorms structures is known to be complex, the models used in this work are reasonably representative of thunderstorms at the southwestern desert of Utah. The electric field models choice of distance from the ground and layer height, is consistent with thunderstorm previously observed at the TASD [1].

Here the CORSIKA package 7.6900 is used to simulate cosmic rays [12]. The secondary particles propagate through the atmosphere and the electric field model implemented. With CORSIKA 7.6900 used in this work both the electromagnetic and the muonic components of the shower are traced through the implemented electric field model until they reach the detector observational 

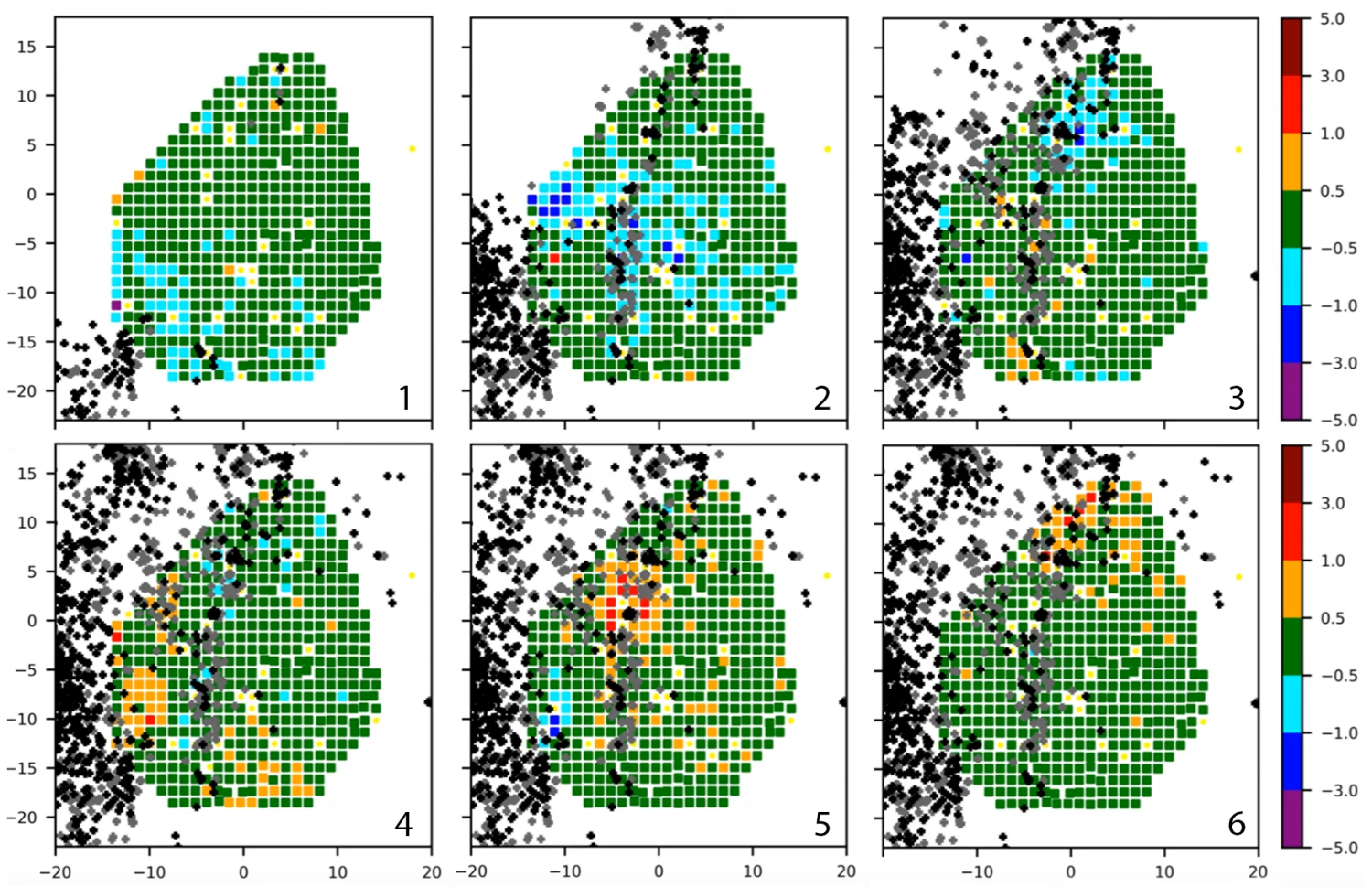

Figure 2: Time evolution of the intensity variation of the secondary shower rate change on the 140927 thunderstorm. Each time frame is 10 minutes.

level. Primary cosmic ray particles were generated between $20 \mathrm{GeV}-10 \mathrm{TeV}$ using SIBYLL [3] for the high energy interaction ( $>80 \mathrm{GeV}$ ) and GHEISHA [10], URQMD [6], and FLUKA [9] for the low energy model $(<80 \mathrm{GeV})$. The zenith and azimuth range from $0^{\circ} \leq \theta \leq 60^{\circ}$ and $0^{\circ} \leq \phi \leq 360^{\circ}$. The energy threshold of secondary particles were set to $0.05 \mathrm{GeV}$ for hadrons, $0.5 \mathrm{GeV}$ for muons, $0.001 \mathrm{GeV}$ for electrons and $0.001 \mathrm{GeV}$ for gammas. Figure 3 shows the distribution of the multiple shower components on the ground at $1400 \mathrm{~m}$ propagated through the atmosphere with no electric field. The simulation was curried out for five simulation sets of electric field ranging from $-2000 \mathrm{~V} / \mathrm{cm}$ to $2000 \mathrm{~V} / \mathrm{cm}$ for each low energy model including a data set with no electric field for background. These events are then propagated through the SD detector following the same trigger condition as the level-0 trigger.

The dependence of the rate variation on the potential difference using URQMD as an example for both thunderstorm model is shown in Figure 4. The direction of the electric field follows CORSIKA's definition, where positive electric field direction is pointing upwards.

\section{Results and conclusion}

Observations by the TASD show that we do observe a deficit and excess in the relative intensity in the secondary cosmic ray shower particles $(\Delta N / N)$ that is mostly between $\pm 1-2 \%$. The simulation results in Figure 4 using SYBILL and URQMD show that the interpretation of $(\Delta N / N)$ is dependent on the model used, the potential difference in the model, and the polarity of that model. 
Particle's Distribution at $1400 \mathrm{~m}-\mathrm{E}$-field $=0$

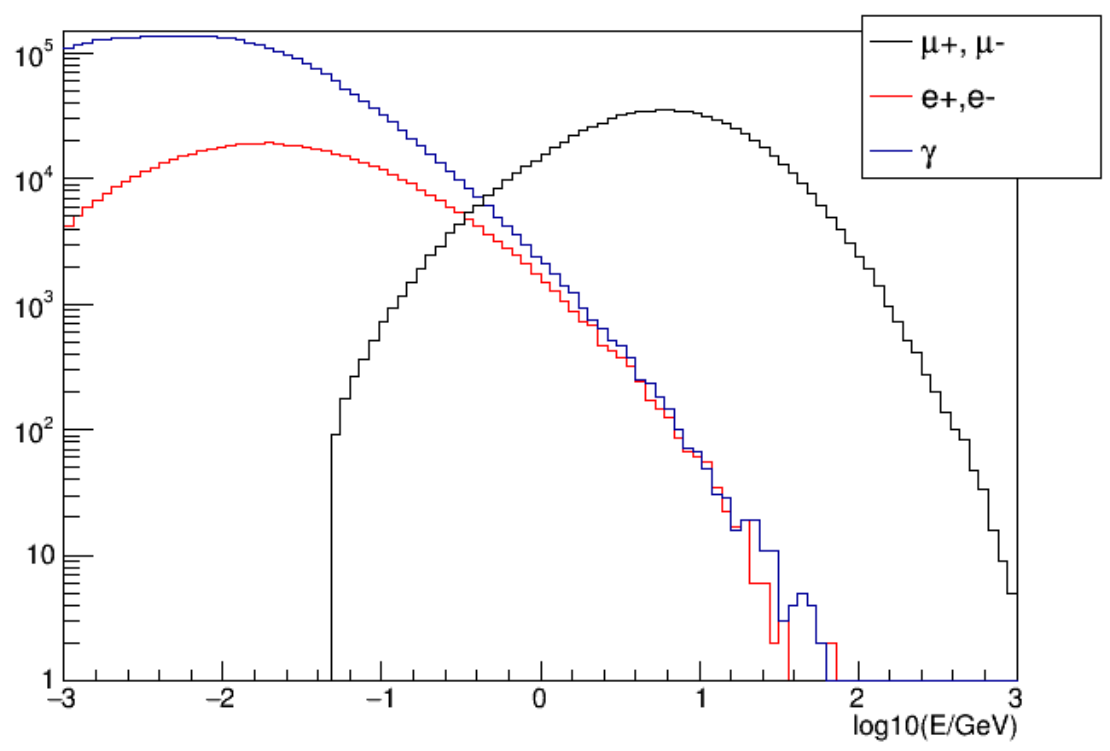

Figure 3: The distributions of particles at $1400 \mathrm{~m}$. The energy threshold of secondary particles were set to $0.05 \mathrm{GeV}$ for hadrons, $0.5 \mathrm{GeV}$ for muons, $0.001 \mathrm{GeV}$ for electrons and $0.001 \mathrm{GeV}$ for gammas. Detector response is not included in this distribution.

URQMD Model-I

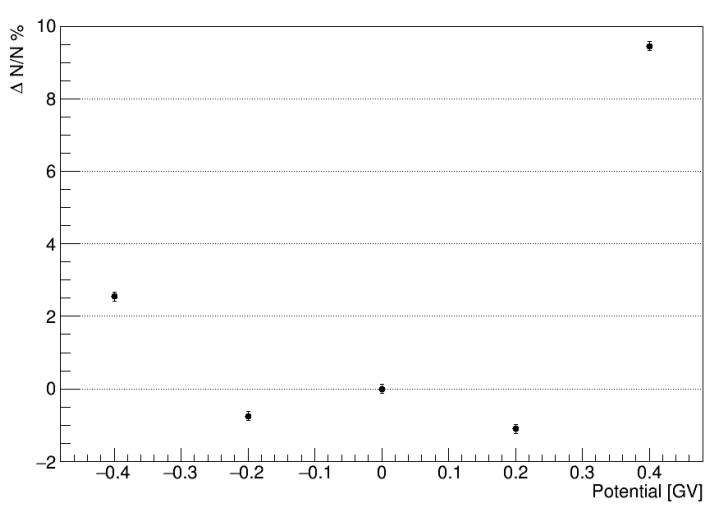

URQMD Model II

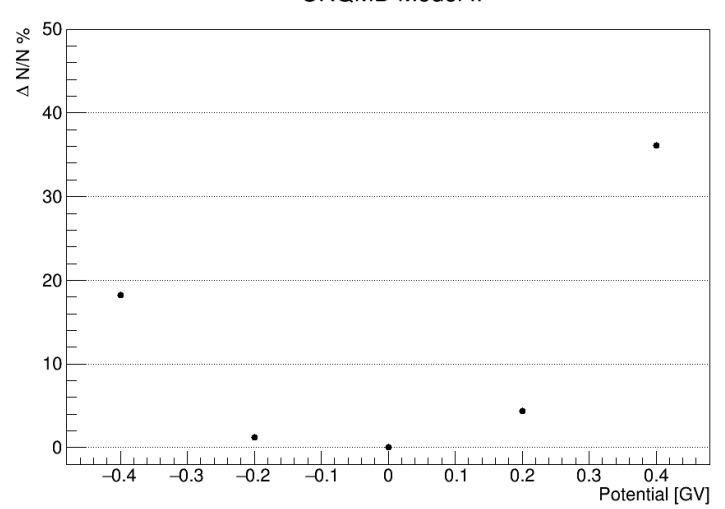

Figure 4: Left: $\Delta N / N$ vs. $\Delta V$ for the a uniform electric field layer inside a cloud (Model I). Right: $\Delta N / N$ $v$ s. $\Delta V$ for the a uniform electric field layer between the cloud and ground (Model II).

The simulation results shown in Figure 4 using five simulation data sets for each model with a potential difference $(\Delta V)$ that varies between -0.4-0.4 GV. When simulating a one layer model of uniform electric field layer inside a cloud referred to as (Model I), the overall profile shows a preliminary deficit in $(\Delta N / N)$ for potential difference of $|\Delta V|$ around $\pm 0.2 \mathrm{GV}$ followed by an excess as the magnitude of the potential difference increases. On the other hand, the simulation results using a simple uniform electric field between cloud and ground (referred to as Model II) show an overall excess in $(\Delta N / N)$. While Model II can explain the excess observed in $(\Delta N / N)$, 
Model I can both explain the deficit and the excess observed in the collected data. Both models show a clear asymmetry between the positive and the negative electric field inside the thunderstorm effect on $(\Delta N / N)$ as observed by the TASD.

In order to interpret the observations of $(\Delta N / N)$ by the TASD we need both the polarity and the type of the thunderstorm (intra-cloud vs. cloud-to-ground). An array of Electric Field Mills at the Telescope Array will allow us to better understand and model these thunderstorms. Currently, an Electric Field Mill (EFM) remote station have been installed approximately in the middle of the Telescope Array site for testing. This will enable us to study the relation between SD observations and the development of thunderstorm's electric field as it progresses on top of the Telescope Array detector.

\section{References}

[1] Abbasi, R. U. et al. (2018). Gamma ray showers observed at ground level in coincidence with downward lightning leaders. Journal of Geophysical Research: Atmospheres, 123(13):68646879 .

[2] Abu-Zayyad, T. et al. (2012). The surface detector array of the Telescope Array experiment. Nuclear Instruments and Methods in Physics Research A, 689:87-97.

[3] Ahn, E.-J., Engel, R., Gaisser, T. K., Lipari, P., and Stanev, T. (2009). Cosmic ray interaction event generator sibyll 2.1. Phys. Rev. D, 80:094003.

[4] Alexeenko, V. V. et al. (1987). Short Perturbations of Cosmic Ray Intensity and Electric Field in Atmosphere. In International Cosmic Ray Conference, volume 4 of International Cosmic Ray Conference, page 272.

[5] Bartoli, B. et al. (2018). Observation of the thunderstorm-related ground cosmic ray flux variations by ARGO-YBJ. Phys. Rev. D, 97(4):042001.

[6] Bass, S. et al. (1998). Microscopic models for ultrarelativistic heavy ion collisions. Progress in Particle and Nuclear Physics, 41:255-369.

[7] C.T.R. Wilson (1956). In Proc. R. Soc. Lond. A, volume 236, page 297.

[8] Eastop Collaboration (2001). The EAS counting rate during thunderstorms. In International Cosmic Ray Conference, volume 10 of International Cosmic Ray Conference, page 4165.

[9] Fasso, A. et al. (2003). The Physics models of FLUKA: Status and recent developments. eConf, C0303241:MOMT005.

[10] Fesefeldt, H. (1985). Report PITHA-85/02, RWTH Aachen.

[11] Hariharan, B. et al. (2019). Measurement of the electrical properties of a thundercloud through muon imaging by the grapes-3 experiment. Phys. Rev. Lett., 122:105101.

[12] Heck, D. et al. (1998). Report FZKA 6019, Forschungszentrum Karlsruhe. 
[13] Kudela, K. et al. (2017). Correlations between secondary cosmic ray rates and strong electric fields at lomnický štít. Journal of Geophysical Research: Atmospheres, 122(20):10,700-10,710.

[14] Marshall, T. C. and Stolzenburg, M. (2001). Voltages inside and just above thunderstorms. Journal of Geophysical Research: Atmospheres, 106(D5):4757-4768.

[15] Muraki, Y. et al. (2004). Effects of atmospheric electric fields on cosmic rays. Phys. Rev. D, 69:123010.

[16] Stolzenburg, M. et al. (2007). Electric field values observed near lightning flash initiations. Geophysical Research Letters, 34(4).

[17] Tsuchiya, H. et al. (2012). Observation of thundercloud-related gamma rays and neutrons in Tibet. Phys. Rev. D, 85(9):092006.

[18] Winn, W. P. and other (1974). Measurements of electric fields in thunderclouds. Journal of Geophysical Research (1896-1977), 79(12):1761-1767. 


\section{Full Authors List: Telescope Array Collaboration}

R.U. Abbasi ${ }^{1}$, T. Abu-Zayyad ${ }^{1,2}$, M. Allen ${ }^{2}$, Y. Arai ${ }^{3}$, R. Arimura ${ }^{3}$, E. Barcikowski ${ }^{2}$, J.W. Belz ${ }^{2}$, D.R. Bergman ${ }^{2}$, S.A. Blake², I. Buckland ${ }^{2}$, R. $\mathrm{Cady}^{2}$, B.G. Cheon ${ }^{4}$, J. Chiba ${ }^{5}$, M. Chikawa ${ }^{6}$, T. Fujii ${ }^{7}$, K. Fujisue ${ }^{6}$, K. Fujita ${ }^{3}$, R. Fujiwara ${ }^{3}$, M. Fukushima ${ }^{6}$, R. Fukushima ${ }^{3}$, G. Furlich ${ }^{2}$, R. Gonzalez ${ }^{2}$, W. Hanlon ${ }^{2}$, M. Hayashi ${ }^{8}$, N. Hayashida ${ }^{9}$, K. Hibino ${ }^{9}$, R. Higuchi ${ }^{6}$, K. Honda ${ }^{10}$,

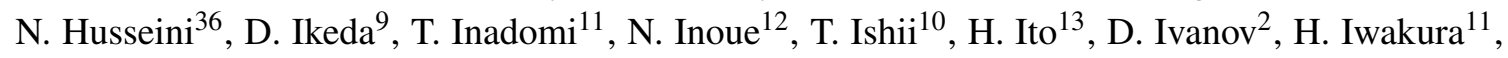
A. Iwasaki ${ }^{3}$, H.M. Jeong ${ }^{14}$, S. Jeong ${ }^{14}$, H. Jhonson ${ }^{1}$, C.C.H. Jui ${ }^{2}$, K. Kadota ${ }^{15}$, F. Kakimoto ${ }^{9}$, O. Kalashev ${ }^{16}$, K. Kasahara ${ }^{17}$, S. Kasami ${ }^{18}$, H. Kawai ${ }^{19}$, S. Kawakami ${ }^{3}$, S. Kawana ${ }^{12}$, K. Kawata ${ }^{6}$, I. Kharuk ${ }^{16}$, E. Kido ${ }^{13}$, H.B. Kim ${ }^{4}$, J.H. Kim ${ }^{2}$, J.H. Kim ${ }^{2}$, M.H. Kim ${ }^{14}$, S.W. Kim ${ }^{14}$, Y. Kimura ${ }^{3}$, S. Kishigami ${ }^{3}$, Y. Kubota ${ }^{11}$, S. Kurisu ${ }^{11}$, V. Kuzmin ${ }^{16^{*}}$, M. Kuznetsov ${ }^{16,20}$, Y.J. Kwon ${ }^{21}$, K.H. Lee ${ }^{14}$, B. Lubsandorzhiev ${ }^{16}$, J.P. Lundquist ${ }^{2,22}$, K. Machida ${ }^{10}$, H. Matsumiya ${ }^{3}$, T. Matsuyama ${ }^{3}$, J.N. Matthews ${ }^{2}$,

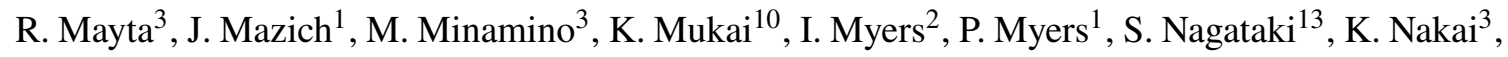
R. Nakamura ${ }^{11}$, T. Nakamura ${ }^{23}$, T. Nakamura ${ }^{11}$, Y. Nakamura ${ }^{11}$, A. Nakazawa ${ }^{11}$, E. Nishio ${ }^{18}$, T. Nonaka ${ }^{6}$, K. O’Brein ${ }^{1}$, H. Oda ${ }^{3}$, S. Ogio ${ }^{3,24}$, M. Ohnishi ${ }^{6}$, H. Ohoka ${ }^{6}$, Y. Oku ${ }^{18}$, T. Okuda ${ }^{25}$, Y. Omura ${ }^{3}$, M. Ono ${ }^{13}$, R. Onogi ${ }^{3}$, A. Oshima ${ }^{3}$, S. Ozawa ${ }^{26}$, I.H. Park ${ }^{14}$, M. Potts ${ }^{2}$, M.S. Pshirkov ${ }^{16,27}$, J. Remington ${ }^{2}$, D.C. Rodriguez ${ }^{2}$, G.I. Rubtsov ${ }^{16}$, D. Ryu ${ }^{28}$, H. Sagawa ${ }^{6}$, R. Sahara ${ }^{3}$, Y. Saito ${ }^{11}$, N. Sakaki ${ }^{6}$, T. Sako ${ }^{6}$, N. Sakurai ${ }^{3}$, K. Sano ${ }^{11}$, K. Sato ${ }^{3}$, T. Seki ${ }^{11}$, K. Sekino ${ }^{6}$, P.D. Shah ${ }^{2}$, Y. Shibasaki ${ }^{11}$, F. Shibata ${ }^{10}$, N. Shibata ${ }^{18}$, T. Shibata ${ }^{6}$, H. Shimodaira ${ }^{6}$, B.K. Shin ${ }^{28}$, H.S. Shin ${ }^{6}$, D. Shinto ${ }^{18}$, J.D. Smith ${ }^{2}$, P. Sokolsky ${ }^{2}$, N. Sone ${ }^{11}$, B.T. Stokes ${ }^{2}$, T.A. Stroman ${ }^{2}$, Y. Takagi ${ }^{3}$,

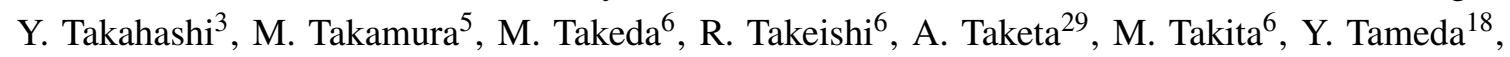
H. Tanaka ${ }^{3}$, K. Tanaka ${ }^{30}$, M. Tanaka ${ }^{31}$, Y. Tanoue ${ }^{3}$, S.B. Thomas ${ }^{2}$, G.B. Thomson ${ }^{2}$, P. Tinyakov ${ }^{16,20}$,

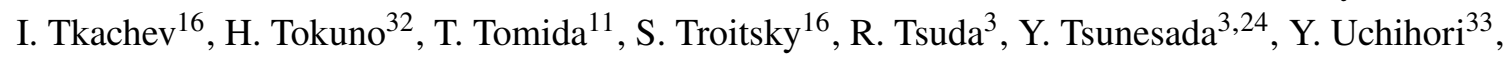
S. Udo ${ }^{9}$, T. Uehama ${ }^{11}$, F. Urban ${ }^{34}$, T. Wong ${ }^{2}$, K. Yada ${ }^{6}$, M. Yamamoto ${ }^{11}$, K. Yamazaki ${ }^{9}$, J. Yang ${ }^{35}$, K. Yashiro ${ }^{5}$, F. Yoshida ${ }^{18}$, Y. Yoshioka ${ }^{11}$, Y. Zhezher ${ }^{6,16}$, and Z. Zundel ${ }^{2}$

${ }^{1}$ Department of Physics, Loyola University Chicago, Chicago, Illinois, USA

${ }^{2}$ High Energy Astrophysics Institute and Department of Physics and Astronomy, University of Utah, Salt Lake City, Utah, USA

${ }^{3}$ Graduate School of Science, Osaka City University, Osaka, Osaka, Japan

${ }^{4}$ Department of Physics and The Research Institute of Natural Science, Hanyang University, Seongdong-gu, Seoul, Korea

${ }^{5}$ Department of Physics, Tokyo University of Science, Noda, Chiba, Japan

${ }^{6}$ Institute for Cosmic Ray Research, University of Tokyo, Kashiwa, Chiba, Japan

7 The Hakubi Center for Advanced Research and Graduate School of Science, Kyoto University, KitashirakawaOiwakecho, Sakyo-ku, Kyoto, Japan

${ }^{8}$ Information Engineering Graduate School of Science and Technology, Shinshu University, Nagano, Nagano, Japan

${ }^{9}$ Faculty of Engineering, Kanagawa University, Yokohama, Kanagawa, Japan

${ }^{10}$ Interdisciplinary Graduate School of Medicine and Engineering, University of Yamanashi, Kofu, Yamanashi, Japan

${ }^{11}$ Academic Assembly School of Science and Technology Institute of Engineering, Shinshu University, Nagano, Nagano, Japan

${ }^{12}$ The Graduate School of Science and Engineering, Saitama University, Saitama, Saitama, Japan

${ }^{13}$ Astrophysical Big Bang Laboratory, RIKEN, Wako, Saitama, Japan

${ }^{14}$ Department of Physics, SungKyunKwan University, Jang-an-gu, Suwon, Korea

${ }^{15}$ Department of Physics, Tokyo City University, Setagaya-ku, Tokyo, Japan

${ }^{16}$ Institute for Nuclear Research of the Russian Academy of Sciences, Moscow, Russia

${ }^{17}$ Faculty of Systems Engineering and Science, Shibaura Institute of Technology, Minato-ku, Tokyo, Japan 
${ }^{18}$ Department of Engineering Science, Faculty of Engineering, Osaka Electro-Communication University, Neyagawashi, Osaka, Japan

${ }^{19}$ Department of Physics, Chiba University, Chiba, Chiba, Japan

${ }^{20}$ Service de Physique Théorique, Université Libre de Bruxelles, Brussels, Belgium

${ }^{21}$ Department of Physics, Yonsei University, Seodaemun-gu, Seoul, Korea

22 Center for Astrophysics and Cosmology, University of Nova Gorica, Nova Gorica, Slovenia

${ }^{23}$ Faculty of Science, Kochi University, Kochi, Kochi, Japan

${ }^{24}$ Nambu Yoichiro Institute of Theoretical and Experimental Physics, Osaka City University, Osaka, Osaka, Japan

${ }^{25}$ Department of Physical Sciences, Ritsumeikan University, Kusatsu, Shiga, Japan

${ }^{26}$ Quantum ICT Advanced Development Center, National Institute for Information and Communications Technology, Koganei, Tokyo, Japan

27 Sternberg Astronomical Institute, Moscow M.V. Lomonosov State University, Moscow, Russia

${ }^{28}$ Department of Physics, School of Natural Sciences, Ulsan National Institute of Science and Technology, UNIST-gil, Ulsan, Korea

${ }^{29}$ Earthquake Research Institute, University of Tokyo, Bunkyo-ku, Tokyo, Japan

${ }^{30}$ Graduate School of Information Sciences, Hiroshima City University, Hiroshima, Hiroshima, Japan

${ }^{31}$ Institute of Particle and Nuclear Studies, KEK, Tsukuba, Ibaraki, Japan

${ }^{32}$ Graduate School of Science and Engineering, Tokyo Institute of Technology, Meguro, Tokyo, Japan

${ }^{33}$ Department of Research Planning and Promotion, Quantum Medical Science Directorate, National Institutes for Quantum and Radiological Science and Technology, Chiba, Chiba, Japan

${ }^{34}$ CEICO, Institute of Physics, Czech Academy of Sciences, Prague, Czech Republic

${ }^{35}$ Department of Physics and Institute for the Early Universe, Ewha Womans University, Seodaaemun-gu, Seoul, Korea

36 J.B. Conant High School, Hoffman Estates, IL, USA.

\section{Acknowledgements}

Operation and analyses of this study have been supported by NSF grants AGS-1844306 and AGS-2112709. The Telescope Array experiment is supported by the Japan Society for the Promotion of Science(JSPS) through Grants-in-Aid for Priority Area 431, for Specially Promoted Research JP21000002, for Scientific Research (S) JP19104006, for Specially Promoted Research JP15H05693, for Scientific Research (S) JP15H05741 and JP19H05607, for Science Research (A) JP18H03705, for Young Scientists (A) JPH26707011, and for Fostering Joint International Research (B) JP19KK0074, by the joint research program of the Institute for Cosmic Ray Research (ICRR), The University of Tokyo; by the Pioneering Program of RIKEN for the Evolution of Matter in the Universe (r-EMU); by the U.S. National Science Foundation awards PHY-1404495, PHY-1404502, PHY-1607727, PHY-1712517, PHY-1806797 and PHY-2012934; by the National Research Foundation of Korea (2017K1A4A3015188, 2020R1A2C1008230, \& 2020R1A2C2102800) ; by the Ministry of Science and Higher Education of the Russian Federation under the contract 075-152020-778, RFBR grant 20-02-00625a (INR), IISN project No. 4.4501.18, and Belgian Science Policy under IUAP VII/37 (ULB). This work was partially supported by the grants ofThe joint research program of the Institute for Space-Earth Environmental Research, Nagoya University and Inter-University Research Program of the Institute for Cosmic Ray Research of University of Tokyo. The foundations of Dr. Ezekiel R. and Edna Wattis Dumke, Willard L. Eccles, and George S. and Dolores Doré Eccles all helped with generous donations. The State of Utah supported the project

\footnotetext{
${ }^{*}$ Deceased
} 
through its Economic Development Board, and the University of Utah through the Office of the Vice President for Research. The experimental site became available through the cooperation of the Utah School and Institutional Trust Lands Administration (SITLA), U.S. Bureau of Land Management (BLM), and the U.S. Air Force. We appreciate the assistance of the State of Utah and Fillmore offices of the BLM in crafting the Plan of Development for the site. Patrick A. Shea assisted the collaboration with valuable advice and supported the collaboration's efforts. The people and the officials of Millard County, Utah have been a source of steadfast and warm support for our work which we greatly appreciate. We are indebted to the Millard County Road Department for their efforts to maintain and clear the roads which get us to our sites. We gratefully acknowledge the contribution from the technical staffs of our home institutions. An allocation of computer time from the Center for High Performance Computing at the University of Utah is gratefully acknowledged. We thank Ryan Said and W. A. Brooks of Vaisala Inc. for providing quality NLDN data lightning discharges over and around the TASD under their academic research use policy. 